\title{
Septic arthritis complicating apatite associated destructive arthropathy
}

\author{
Adrian Jones, M John Henderson, Peter Berman, Michael Doherty
}

\begin{abstract}
Septic arthritis can complicate many forms of arthritis. Two cases of apatite associated destructive arthropathy (AADA) complicated by sepsis are described. Diagnosis of this complication in the setting of severe joint damage is difficult as AADA and sepsis share certain characteristics-an initial, rapidly progressive, severely painful course and radiographs which show rapidly destructive changes with marked cartilage loss, bone attrition, and virtual absence of osteophyte or cyst response.
\end{abstract}

Apatite associated destructive arthritis (AADA) is an increasingly recognised rapidly progressive subset of osteoarthritis, showing marked predilection for the elderly. Although first emphasised at the shoulder ('Milwaukee shoulder syndrome' ${ }^{\text {, }}$, it subsequently has been described in other large joints, including the hip ('analgesic hip ${ }^{2-5}$ ). Clinically the condition is characterised by severe usage, rest and night pain, instability, large cool effusions, and 'noninflammatory' synovial fluid containing plentiful aggregates of apatite (basic calcium phosphate). Radiographs show rapidly destructive change with marked cartilage and bone attrition, a paucity of osteophytic and new bone response, often apparent widening of joint space, and 'pressure erosion' of adjacent cortical bone; progressive protrusio acetabuli may develop at the hip. Because of these radiographic features and rapid progression the main differential diagnoses to consider are atrophic Charcot arthropathy, avascular necrosis, and sepsis. ${ }^{3}$

We report two cases of AADA of the hip complicated by joint sepsis.

\section{Case reports}

CASE 1

A 79 year old woman was referred with a nine month history of right hip pain unassociated with antecedent trauma. For five years she had taken naproxen $250 \mathrm{mg}$ twice a day for symptomatic hand interphalangeal osteoarthritis; she also took prednisolone $7.5 \mathrm{mg}$ daily for fibrosing alveolitis (well controlled since diagnosis four years before) and diuretics for chronic leg oedema. On examination her right hip was tender, with painful restriction of movement in a capsular pattern; she also had polyarticular interphalangeal hand osteoarthritis and wrist disease consistent with pyrophosphate arthropathy. Apart from fine inspiratory crackles at both lung bases and bilateral leg oedema the remainder of the examination was normal.

Right hip radiographs showed marked osteo- arthritic changes-decrease in interosseous distance, cysts, sclerosis, osteophyte-in a superolateral pattern (fig 1A), with loss of height and lateral subluxation of the right femoral head. Bone density was well preserved and the appearance was that of severe uncomplicated osteoarthritis. Mild osteoarthritic changes were present in the left hip, numerous interphalangeal joints, and both scaphotrapezoid joints. Chondrocalcinosis was noted in the symphysis pubis, wrists, and knees.

She was placed on the waiting list for arthroplasty, but four months later was admitted with right middle lobe pneumonia, rapid atrial fibrillation, and pulmonary oedema. This was successfully treated with pivampicillin, digoxin, and an increase in diuretics. During convalescence, however, she complained of increasing pain in her right hip. Hip radiographs at this time (fig 1B) showed marked attrition of the femoral head and acetabulum, modest sclerosis with minimal osteophyte, and retained bone density, consistent with a diagnosis of AADA. Five days later she developed pain and swelling around the medial aspect of the right knee, where an ill defined, tender, warm swelling was palpable above, and extending over, the right medial joint line. Aspiration yielded only a few drops of clear fluid and ultrasound examination showed diffuse thickening of the medial aspect of the knee with no clearly delineated boundaries. Injection of lignocaine into the tender area, presumed to be a medial fat pad, gave good symptomatic relief. Two weeks later, however, recurrence of right medial knee pain, associated with fever and general deterioration, led to repeat aspiration of the tender swelling. Bloodstained fluid (1 ml) was obtained, from which no organism was isolated. There was no other apparent focus of infection (chest radiograph was now clear), but blood culture isolated penicillin resistant Staphylococcus aureus. She was given intravenous flucloxacillin but rapidly deteriorated and died.

At necropsy the right hip showed marked loss of cartilage and bone affecting both femoral and acetabular components, and purulent synovial fluid. In addition, a communicating cavity, up to $4 \mathrm{~cm}$ in diameter, extended from the hip joint throughout the anterolateral aspect of the thigh. Death was attributed to $S$ aureus septicaemia associated with septic arthritis in a hip already affected by AADA.

\section{CASE 2}

A 74 year old man was admitted after a fall. Two years previously he described rapid onset of pain in both hips; since when he had needed 
Figure 1 Right hip radiographs of case 1: (A) initial film showing superolateral osteoarthritis; (B) film taken four months later, showing marked attrition of femoral head and acetabulum, modest sclerosis, calcific debris, and retained bone density, consistent with progression to apatite associated destructive arthritis.
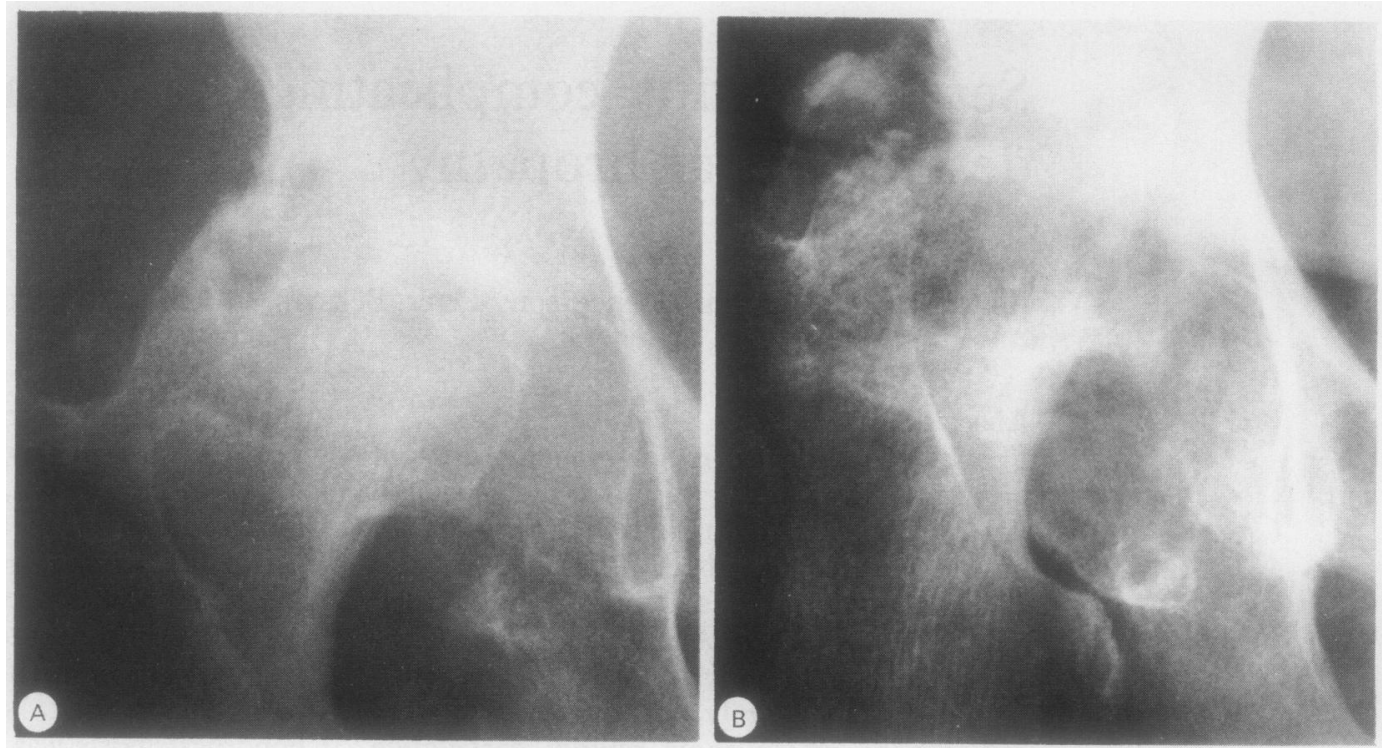

Figure 2 Right $(A)$ and left $(B)$ hip radiographs of case 2, showing bilateral severe destructive arthropathy characterised by marked attrition of femoral and acetabular bone, apparent joint space widening, modest sclerosis, no osteophyte, and superolateral migration consistent with apatite associated destructive arthritis.
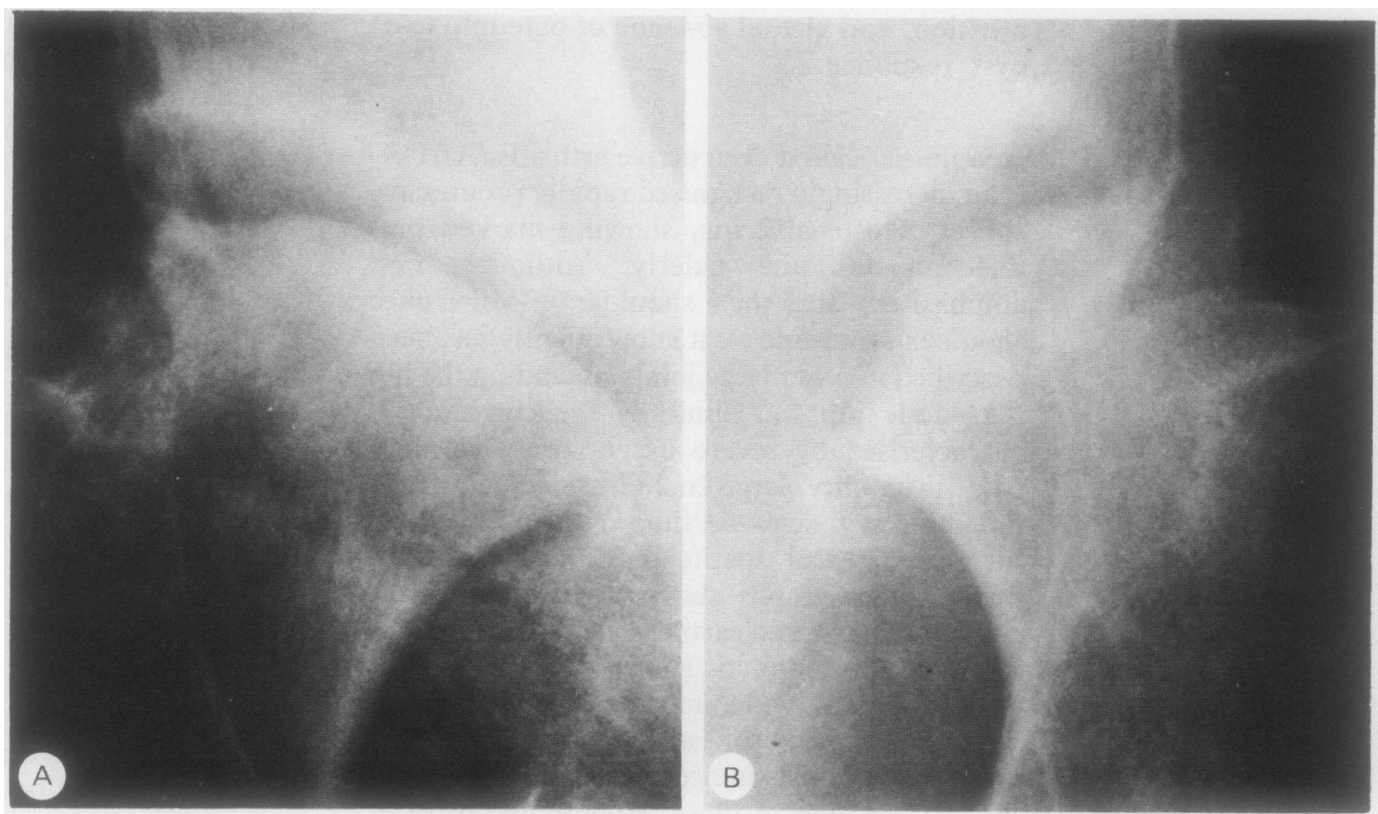

sticks for walking. He had also had more longstanding but less severe symptoms in both wrists, right shoulder, and left midfoot. He had taken only co-proxamol for joint symptoms. On admission he complained of increase in his usual right hip pain, but otherwise felt well. Fourteen years previously he had undergone successful drainage of a liver abscess (no microbiological details available).

General examination was unremarkable and he had no fever. Both hips showed crepitus, abductor weakness, but only modest restriction of movement: his right hip was tender over the anterior joint line, and he showed a marked Trendelenburg gait. In addition, he had clinical evidence of mild osteoarthritic changes in symptomatic joints in a distribution consistent with pyrophosphate arthropathy.

Investigations included white cell count $20000 \times 10^{9} / 1$ (neutrophils $17 \cdot 8 \times 10^{9} / 1$ ), erythrocyte sedimentation rate $104 \mathrm{~mm} / \mathrm{h}$, alkaline phosphatase $416 \mathrm{IU} / 1$ (normal 80-200), and $\gamma$ glutamyltransferase $127 \mathrm{IU} / 1$ (normal 10-50).
Hip radiographs (supine films, fig 2) showed bilateral severe destructive disease with marked attrition of bone, modest sclerosis, no osteophyte, superolateral migration (particularly on the left), and apparent joint space widening consistent with AADA. Other symptomatic joints showed hypertrophic osteoarthritic changes typical of pyrophosphate arthropathy, and chondrocalcinosis in knees and right wrist.

One week after admission he developed low grade fever and night sweats, and complained of increasing pain in the left hip. Examination showed a tender swelling in the left groin extending down the anteromedial thigh. Hip arthrography confirmed that this swelling extended from the joint, with leakage of contrast both inferomedially and superiorly. Streptococcus milleri was cultured from the hip aspirate. He was treated with intravenous benzylpenicillin with some clinical improvement. Four days later, however, he collapsed and died from an apparent pulmonary embolus. Permission for necropsy was refused. 


\section{Discussion}

In both patients clinical and radiographic features of hip disease were consistent with a diagnosis of AADA. ${ }^{35}$ The relation between this condition and non-steroidal anti-inflammatory drug (NSAID) usage remains controversial, ${ }^{5}$ and it is perhaps noteworthy that one of the two patients had not received such drugs. Interestingly, in addition to AADA at the hip, both patients had chondrocalcinosis and pyrophosphate arthropathy at other sites, an unexplained association emphasised in previous cases of rapidly destructive hip disease. ${ }^{6}$ In both patients the time course of symptom exacerbation, fever, and systemic upset, and the presence of severe destructive change without osteopenia or more characteristic radiographic features of infection suggest that septic arthritis was a late secondary complication of pre-existing AADA. In the first case chest infection preceded the episode of hip sepsis, but in the second no primary focus was identified, though the nature of the organism (Strep milleri) suggests a gastrointestinal/buccal origin. ${ }^{7}$ The expected painful nature and radiographic appearance of AADA presented difficulties in early recognition of the additional sepsis, particularly in the first case. Despite eventual diagnosis and treatment for septic arthritis both patients died.

As far as we are aware septic arthritis has not previously been described as a complication of AADA, though it is well described in association with other abnormal joints, ${ }^{8}$ including crystal associated arthropathy ${ }^{10}$ and osteonecrosis. ${ }^{11}$ As illustrated in these two cases, however, certain shared characteristics of AADA and sepsis may present particular problems in diagnosing dual disease. For example, AADA, like sepsis, often follows an initial, rapidly progressive, severely painful course, and radiographs show rapidly destructive changes with marked cartilage loss, bone attrition, and virtual absence of osteophyte or cyst response. Sepsis, osteonecrosis, and atrophic Charcot arthropathy are the principal differential diagnoses of AADA. ${ }^{3}$ Radiographic features differentiating AADA from sepsis include absence of osteopenia, erosions, and periostitis: such changes, however, may take several weeks to appear, and no such changes were present in our two cases. Furthermore, with such an abnormal partially destroyed joint as the starting point, such changes, particularly cortical erosion, would probably be detected only with great difficulty, especially in recent onset sepsis. Clinically, of course, evidence of systemic upset with fever and raised erythrocyte sedimentation rate should alert one to the possibility of septic arthritis, and these features were important in determining further investigation in our patients.

A further clinical feature of interest in both cases was the presence of joint 'cysts' extending from the affected hip into the anteromedial aspect of the thigh. This was correctly recognised in the second case, but was misinterpreted in the first patient because of presentation as a distant ill defined swelling (superior to the medial joint line of the knee) without clinical evidence of intervening communication. Although large, cool effusions are characteristic of AADA affecting the shoulder or knee, ${ }^{134}$ these have not been emphasised at the hip. Possibly, therefore, gross symptomatic hip effusions that track or leak into the thigh may be clinical features particularly associated with sepsis rather than AADA, and if present should lead to joint aspiration.

Early diagnosis of joint sepsis always requires increased vigilance. Both these cases exemplify a previous warning: '.. when an elderly patient complains of pain or swelling of a joint, the conclusion is too readily made that he has a non-infectious disease of the involved joint'.8

MD gratefully acknowledges the financial support of the Arthritis and Rheumatism Council.

1 McCarty D J, Halverson P B, Carrera G F, Brewer B J, Kozin F. 'Milwaukee shoulder'-2ssociation of microKozin F. Milwaukee shoulder'-association of microspheroids containing hydroxyapatite crystals, active col-
lagenase, and neutral protease with rotator cuff defects. lagenase, and neutral protease with
Arthritis Rhewim 1981; 24: 464-73.

2 Schumacher H R, Somlyo A P, Tse R L, Mauret K. Arthritis associated with apatite crystals. Amn Intern Med 1977; 87: 411-6.

3 Dieppe P A, Doherty M, MacFarlane D G, Hutton C W Bradfield J W, Watt I. Apatite-associated destructive arthritis. Br $\mathcal{F}$ Rhewematol 1984; 23: 84-91.

4 Halverson P B, McCarty D J, Cheung H S, Ryan L M. Milwaukee shoulder syndrome: eleven additional cases Milwaukee shoulder syndrome: eleven additional cases with involvement of the knee in seven (basic calcium phosphate crystal deposid

5 Doherty M, Holt M, MacMillan P, Watt I, Dieppe P. A reappraisal of 'analgesic hip'. Ann Rhewn' Dis 1986; 45: reappras

6 Menkes C J, Decraemere W, Postel M, Forest M. Chondrocalcinosis and rapid destruction of the hip. $\mathcal{F}$ Rheumatol 1985; 12: 130-3.

7 Murray H W, Gross K C, Masur H, Roberts R B. Serious infections caused by Streptococcus milleri. Am $\mathcal{f}$ Med 1978 64: 759-64.

8 Kelly P J, Martin W J, Coventry M B. Bacterial (suppurative) arthritis in the adult. $\mathcal{f}$ Bome foint Surg [Am] 1970; 52: $1595-602$.

9 Mayer J W, De Horatius R J, Messner R P. Serratia marcescens-caused arthritis with negative and positive marcescens-caused arthritis with negative and positive
birefringent crystals. Arch Intem Med 1976; 136: 1323-5.

10 Zyskowski L P, Silverfield J C, O'Duffy D. Pseudogout

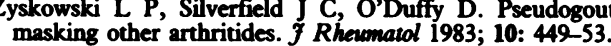

11 Phillips F M, Pottenger L A. Acute septic arthritis in chronic osteonecrosis of the hip. $\mathcal{I}$ Rheumatol 1988; 15: 1713-6. 